\title{
Strong Convergence Algorithm for Split Equilibrium Problems and Hierarchical Fixed Point Problems
}

\author{
Abdellah Bnouhachem ${ }^{1,2}$ \\ ${ }^{1}$ School of Management Science and Engineering, Nanjing University, Nanjing 210093, China \\ ${ }^{2}$ Ibn Zohr University, ENSA, BP 1136, Agadir, Morocco
}

Correspondence should be addressed to Abdellah Bnouhachem; babedallah@yahoo.com

Received 24 August 2013; Accepted 23 December 2013; Published 20 February 2014

Academic Editors: F.-S. Hsieh, K. R. Kazmi, N. Petrot, and D. Xu

Copyright (C) 2014 Abdellah Bnouhachem. This is an open access article distributed under the Creative Commons Attribution License, which permits unrestricted use, distribution, and reproduction in any medium, provided the original work is properly cited.

The purpose of this paper is to investigate the problem of finding the approximate element of the common set of solutions of a split equilibrium problem and a hierarchical fixed point problem in a real Hilbert space. We establish the strong convergence of the proposed method under some mild conditions. Several special cases are also discussed. Our main result extends and improves some well-known results in the literature.

\section{Introduction}

Let $H$ be a real Hilbert space, whose inner product and norm are denoted by $\langle\cdot, \cdot\rangle$ and $\|\cdot\|$. Let $C$ be a nonempty closed convex subset of $H$. We introduce the following definitions which are useful in the following analysis.

Definition 1. The mapping T: $C \rightarrow H$ is said to be

(a) monotone, if

$$
\langle T x-T y, x-y\rangle \geq 0, \quad \forall x, y \in C ;
$$

(b) strongly monotone, if there exists $\alpha>0$ such that

$$
\langle T x-T y, x-y\rangle \geq \alpha\|x-y\|^{2}, \quad \forall x, y \in C ;
$$

(c) $\alpha$-inverse strongly monotone, if there exists $\alpha>0$ such that

$$
\langle T x-T y, x-y\rangle \geq \alpha\|T x-T y\|^{2}, \quad \forall x, y \in C ;
$$

(d) nonexpansive, if

$$
\|T x-T y\| \leq\|x-y\|, \quad \forall x, y \in C ;
$$

(e) $k$-Lipschitz continuous, if there exists a constant $k>0$ such that

$$
\|T x-T y\| \leq k\|x-y\|, \quad \forall x, y \in C ;
$$

(f) contraction on $C$, if there exists a constant $0 \leq k<1$ such that

$$
\|T x-T y\| \leq k\|x-y\|, \quad \forall x, y \in C .
$$

It is easy to observe that every $\alpha$-inverse strongly monotone $T$ is monotone and Lipschitz continuous. It is well known that every nonexpansive operator $T: H \rightarrow H$ satisfies, for all $(x, y) \in H \times H$, the inequality

$$
\begin{gathered}
\langle(x-T(x))-(y-T(y)), T(y)-T(x)\rangle \\
\leq \frac{1}{2}\|(T(x)-x)-(T(y)-y)\|^{2}
\end{gathered}
$$

and therefore, we get, for all $(x, y) \in H \times \operatorname{Fix}(T)$,

$$
\langle x-T(x), y-T(x)\rangle \leq \frac{1}{2}\|T(x)-x\|^{2} .
$$

See, for example, [1, Theorem 1], and [2, Theorem 3].

The fixed point problem for the mapping $T$ is to find $x \in C$ such that

$$
T x=x .
$$

We denote by $F(T)$ the set of solutions of (9). It is well known that $F(T)$ is closed and convex and $P_{F}(T)$ is well defined (see [3]). 
The equilibrium problem denoted by EP is to find $x \in C$ such that

$$
F(x, y) \geq 0, \quad \forall y \in C .
$$

The solution set of (10) is denoted by $\operatorname{EP}(F)$. Numerous problems in physics, optimization, and economics reduce to finding a solution of (10); see [4-7]. In 1997, Combettes and Hirstoaga [8] introduced an iterative scheme of finding the best approximation to the initial data when $\operatorname{EP}(F)$ is nonempty. In 2007, Plubtieng and Punpaeng [6] introduced an iterative method for finding the common element of the set $F(T) \cap \operatorname{EP}(F)$.

Recently, Censor et al. [9] introduced a new variational inequality problem which we call the split variational inequality problem (SVIP). Let $H_{1}$ and $H_{2}$ be two real Hilbert spaces. Given operators $f: H_{1} \rightarrow H_{1}$ and $g: H_{2} \rightarrow H_{2}$, a bounded linear operator $A: H_{1} \rightarrow H_{2}$, and nonempty, closed, and convex subsets $C \subseteq H_{1}$ and $Q \subseteq H_{2}$, the SVIP is formulated as follows: find a point $x^{*} \in C$ such that

$$
\left\langle f\left(x^{*}\right), x-x^{*}\right\rangle \geq 0 \quad \forall x \in C
$$

and such that

$$
\begin{array}{r}
y^{*}=A x^{*} \in Q \text { solves }\left\langle g\left(y^{*}\right), y-y^{*}\right\rangle \geq 0 \\
\forall y \in Q .
\end{array}
$$

In [10], Moudafi introduced an iterative method which can be regarded as an extension of the method given by Censor et al. [9] for the following split monotone variational inclusions:

$$
\text { Find } x^{*} \in H_{1} \text { such that } 0 \in f\left(x^{*}\right)+B_{1}\left(x^{*}\right)
$$

and such that

$$
y^{*}=A x^{*} \in H_{2} \text { solves } 0 \in g\left(y^{*}\right)+B_{2}\left(y^{*}\right),
$$

where $B_{i}: H_{i} \rightarrow 2^{H_{i}}$ is a set-valued mapping for $i=1$, 2. Later Byrne et al. [11] generalized and extended the work of Censor et al. [9] and Moudafi [10].

Very recently, Kazmi and Rizvi [12] studied the following pair of equilibrium problems called split equilibrium problem: let $F_{1}: C \times C \rightarrow R$ and $F_{2}: Q \times Q \rightarrow R$ be nonlinear bifunctions and let $A: H_{1} \rightarrow H_{2}$ be a bounded linear operator; then, the split equilibrium problem (SEP) is to find $x^{*} \in$ $C$ such that

$$
F_{1}\left(x^{*}, x\right) \geq 0, \quad \forall x \in C
$$

and such that

$$
y^{*}=A x^{*} \in Q \text { solves } F_{2}\left(y^{*}, y\right) \geq 0, \quad \forall y \in Q .
$$

The solution set of SEP (15)-(16) is denoted by $\Lambda=\{p \in$ $\left.\operatorname{EP}\left(F_{1}\right): A p \in \operatorname{EP}\left(F_{2}\right)\right\}$.

Let $S: C \rightarrow H$ be a nonexpansive mapping. The following problem is called a hierarchical fixed point problem: find $x \in$ $F(T)$ such that

$$
\langle x-S x, y-x\rangle \geq 0, \quad \forall y \in F(T) .
$$

It is known that the hierarchical fixed point problem (17) links with some monotone variational inequalities and convex programming problems; see $[13,14]$. Various methods [1520] have been proposed to solve the hierarchical fixed point problem. In 2010, Yao et al. [14] introduced the following strong convergence iterative algorithm to solve the problem (17):

$$
\begin{gathered}
y_{n}=\beta_{n} S x_{n}+\left(1-\beta_{n}\right) x_{n}, \\
x_{n+1}=P_{C}\left[\alpha_{n} f\left(x_{n}\right)+\left(1-\alpha_{n}\right) T y_{n}\right], \quad \forall n \geq 0,
\end{gathered}
$$

where $f: C \rightarrow H$ is a contraction mapping and $\left\{\alpha_{n}\right\}$ and $\left\{\beta_{n}\right\}$ are two sequences in $(0,1)$. Under some certain restrictions on parameters, Yao et al. proved that the sequence $\left\{x_{n}\right\}$ generated by (18) converges strongly to $z \in F(T)$, which is the unique solution of the following variational inequality:

$$
\langle(I-f) z, y-z\rangle \geq 0, \quad \forall y \in F(T) .
$$

In 2011, Ceng et al. [21] investigated the following iterative method:

$$
x_{n+1}=P_{C}\left[\alpha_{n} \rho U\left(x_{n}\right)+\left(I-\alpha_{n} \mu F\right)\left(T\left(y_{n}\right)\right)\right], \quad \forall n \geq 0,
$$

where $U$ is a Lipschitzian mapping and $F$ is a Lipschitzian and strongly monotone mapping. They proved that under some approximate assumptions on the operators and parameters, the sequence $\left\{x_{n}\right\}$ generated by (20) converges strongly to the unique solution of the variational inequality

$$
\langle\rho U(z)-\mu F(z), x-z\rangle \geq 0, \quad \forall x \in \operatorname{Fix}(T) .
$$

In the present paper, inspired by the above cited works and by the recent works going in this direction, we give an iterative method for finding the approximate element of the common set of solutions of (15)-(16) and (17) in real Hilbert space. Strong convergence of the iterative algorithm is obtained in the framework of Hilbert space. We would like to mention that our proposed method is quite general and flexible and includes many known results for solving split equilibrium problems and hierarchical fixed point problems; see, for example, [13, 14, 17-19, 21-23] and relevant references cited therein.

\section{Preliminaries}

In this section, we recall some basic definitions and properties, which will be frequently used in our later analysis. Some useful results proved already in the literature are also summarized. The first lemma provides some basic properties of projection onto $C$.

Lemma 2. Let $P_{C}$ denote the projection of $H$ onto $C$. Then, one has the following inequalities:

$$
\begin{gathered}
\left\langle z-P_{C}[z], P_{C}[z]-v\right\rangle \geq 0, \quad \forall z \in H, v \in C ; \\
\left\langle u-v, P_{C}[u]-P_{C}[v]\right\rangle \geq\left\|P_{C}[u]-P_{C}[v]\right\|^{2}, \quad \forall u, v \in H ; \\
\left\|P_{C}[u]-P_{C}[v]\right\| \leq\|u-v\|, \quad \forall u, v \in H ; \\
\left\|u-P_{C}[z]\right\|^{2} \leq\|z-u\|^{2}-\left\|z-P_{C}[z]\right\|^{2}, \quad \forall z \in H, u \in C .
\end{gathered}
$$


Assumption 3 (see [24]). Let $F: C \times C \rightarrow \mathbb{R}$ be a bifunction satisfying the following assumptions:

(i) $F(x, x)=0$, for all $x \in C$;

(ii) $F$ is monotone; that is, $F(x, y)+F(y, x) \leq 0$, for all $x, y \in C$;

(iii) for each $x, y, z \in C, \lim _{t \rightarrow 0} F(t z+(1-t) x, y) \leq F(x, y)$;

(iv) for each $x \in C, y \rightarrow F(x, y)$ is convex and lower semicontinuous;

(v) for fixed $r>0$ and $z \in C$, there exists a bounded subset $K$ of $H_{1}$ and $x \in C \cap K$ such that

$$
F(y, x)+\frac{1}{r}\langle y-x, x-z\rangle \geq 0, \quad \forall y \in C \backslash K
$$

Lemma 4 (see [8]). Assume that $F_{1}: C \times C \rightarrow \mathbb{R}$ satisfies Assumption 3. For $r>0$ and for all $x \in H_{1}$, define a mapping $T_{r}^{F_{1}}: H_{1} \rightarrow C$ as follows:

$$
\begin{gathered}
T_{r}^{F_{1}}(x)=\left\{z \in C: F_{1}(z, y)+\frac{1}{r}\langle y-z, z-x\rangle \geq 0,\right. \\
\forall y \in C\} .
\end{gathered}
$$

Then the following hold:

(i) $T_{r}^{F_{1}}$ is nonempty and single-valued;

(ii) $T_{r}^{F_{1}}$ is firmly nonexpansive; that is,

$$
\begin{array}{r}
\left\|T_{r}^{F_{1}}(x)-T_{r}^{F_{1}}(y)\right\|^{2} \leq\left\langle T_{r}^{F_{1}}(x)-T_{r}^{F_{1}}(y), x-y\right\rangle, \\
\forall x, y \in H_{1} ;
\end{array}
$$

(iii) $F\left(T_{r}^{F_{1}}\right)=E P\left(F_{1}\right)$;

(iv) $E P\left(F_{1}\right)$ is closed and convex.

Assume that $F_{2}: Q \times Q \rightarrow \mathbb{R}$ satisfies Assumption 3. For $s>0$ and for all $u \in H_{2}$, define a mapping $T_{s}^{F_{2}}: H_{2} \rightarrow Q$ as follows:

$$
\begin{aligned}
T_{s}^{F_{2}}(u)=\{v & \in Q: F_{2}(v, w)+\frac{1}{s}\langle w-v, v-u\rangle \\
& \geq 0, \forall w \in Q\} .
\end{aligned}
$$

Then $T_{s}^{F_{2}}$ satisfies conditions (i)-(iv) of Lemma 4. Consider $F\left(T_{s}^{F_{2}}\right)=\operatorname{EP}\left(F_{2}, Q\right)$, where $\operatorname{EP}\left(F_{2}, Q\right)$ is the solution set of the following equilibrium problem:

$$
\text { find } y^{*} \in Q \text { such that } F_{2}\left(y^{*}, y\right) \geq 0, \quad \forall y \in Q \text {. }
$$

Lemma 5 (see [25]). Assume that $F_{1}: C \times C \rightarrow \mathbb{R}$ satisfies Assumption 3, and let $T_{r}^{F_{1}}$ be defined as in Lemma 4. Let $x, y \in$ $H_{1}$ and $r_{1}, r_{2}>0$. Then

$$
\left\|T_{r_{2}}^{F_{1}}(y)-T_{r_{1}}^{F_{1}}(x)\right\| \leq\|y-x\|+\left|\frac{r_{2}-r_{1}}{r_{2}}\right|\left\|T_{r_{2}}^{F_{1}}(y)-y\right\| .
$$

Lemma 6 (see [26]). Let $C$ be a nonempty closed convex subset of a real Hilbert space $H$. If $T: C \rightarrow C$ is a nonexpansive mapping with $\operatorname{Fix}(T) \neq \emptyset$, then the mapping $I-T$ is demiclosed at 0 ; that is, if $\left\{x_{n}\right\}$ is a sequence in $C$ weakly converging to $x$ and if $\left\{(I-T) x_{n}\right\}$ converges strongly to 0 , then $(I-T) x=0$.

Lemma 7 (see [21]). Let $U: C \rightarrow H$ be $\tau$-Lipschitzian mapping and let $\mathrm{F}: \mathrm{C} \rightarrow \mathrm{H}$ be a $k$-Lipschitzian and $\eta$-strongly monotone mapping; then for $0 \leq \rho \tau<\mu \eta, \mu F-\rho U$ is $\mu \eta-\rho \tau$ strongly monotone; that is,

$$
\begin{array}{r}
\langle(\mu F-\rho U) x-(\mu F-\rho U) y, x-y\rangle \geq(\mu \eta-\rho \tau)\|x-y\|^{2}, \\
\forall x, y \in C .
\end{array}
$$

Lemma 8 (see [27]). Suppose that $\lambda \in(0,1)$ and $\mu>0$. Let $F: C \rightarrow H$ be an $k$-Lipschitzian and $\eta$-strongly monotone operator. In association with nonexpansive mapping $T: C \rightarrow C$, define the mapping $T^{\lambda}: C \rightarrow H$ by

$$
T^{\lambda} x=T x-\lambda \mu F T(x), \quad \forall x \in C .
$$

Then $T^{\lambda}$ is a contraction provided that $\mu<\left(2 \eta / k^{2}\right)$; that is,

$$
\left\|T^{\lambda} x-T^{\lambda} y\right\| \leq(1-\lambda \nu)\|x-y\|, \quad \forall x, y \in C,
$$

where $v=1-\sqrt{1-\mu\left(2 \eta-\mu L^{2}\right)}$.

Lemma 9 (see [28]). Assume that $\left\{a_{n}\right\}$ is a sequence of nonnegative real numbers such that

$$
a_{n+1} \leq\left(1-\gamma_{n}\right) a_{n}+\delta_{n}
$$

where $\left\{\gamma_{n}\right\}$ is a sequence in $(0,1)$ and $\delta_{n}$ is a sequence such that

(1) $\sum_{n=1}^{\infty} \gamma_{n}=\infty$;

(2) $\lim \sup _{n \rightarrow \infty} \delta_{n} / \gamma_{n} \leq 0$ or $\sum_{n=1}^{\infty}\left|\delta_{n}\right|<\infty$.

Then $\lim _{n \rightarrow \infty} a_{n}=0$.

Lemma 10 (see [29]). Let $C$ be a closed convex subset of $H$. Let $\left\{x_{n}\right\}$ be a bounded sequence in $H$. Assume that

(i) the weak $w$-limit set $w_{w}\left(x_{n}\right) \subset C$ where $w_{w}\left(x_{n}\right)=$ $\left\{x: x_{n_{i}} \rightarrow x\right\}$

(ii) for each $z \in C, \lim _{n \rightarrow \infty}\left\|x_{n}-z\right\|$ exists.

Then $\left\{x_{n}\right\}$ is weakly convergent to a point in C.

\section{The Proposed Method and Some Properties}

In this section, we suggest and analyze our method and we prove a strong convergence theorem for finding the common solutions of the split equilibrium problem (15)-(16) and the hierarchical fixed point problem (17).

Let $H_{1}$ and $H_{2}$ be two real Hilbert spaces and let $C \subseteq$ $H_{1}$ and $Q \subseteq H_{2}$ be nonempty closed convex subsets of Hilbert spaces $H_{1}$ and $H_{2}$, respectively. Let $A: H_{1} \rightarrow H_{2}$ be a bounded linear operator. Assume that $F_{1}: C \times C \rightarrow \mathbb{R}$ 
and $F_{2}: Q \times Q \rightarrow \mathbb{R}$ are the bifunctions satisfying Assumption 3 and $F_{2}$ is upper semicontinuous in first argument. Let $S, T: C \rightarrow C$ be a nonexpansive mapping such that $\Lambda \cap$ $F(T) \neq \emptyset$. Let $F: C \rightarrow C$ be an $k$-Lipschitzian mapping and $\eta$-strongly monotone and let $U: C \rightarrow C$ be $\tau$-Lipschitzian mapping. Now we introduce the proposed method as follows.

Algorithm 11. For a given $x_{0} \in C$ arbitrarily, let the iterative sequences $\left\{u_{n}\right\},\left\{x_{n}\right\}$, and $\left\{y_{n}\right\}$ be generated by

$$
\begin{gathered}
u_{n}=T_{r_{n}}^{F_{1}}\left(x_{n}+\gamma A^{*}\left(T_{r_{n}}^{F_{2}}-I\right) A x_{n}\right) ; \\
y_{n}=\beta_{n} S x_{n}+\left(1-\beta_{n}\right) u_{n} ; \\
x_{n+1}=P_{C}\left[\alpha_{n} \rho U\left(x_{n}\right)+\left(I-\alpha_{n} \mu F\right)\left(T\left(y_{n}\right)\right)\right], \quad \forall n \geq 0,
\end{gathered}
$$

where $\left\{r_{n}\right\} \subset(0,2 \varsigma)$ and $\gamma \in(0,1 / L), L$ is the spectral radius of the operator $A^{*} A$, and $A^{*}$ is the adjoint of $A$. Suppose that the parameters satisfy $0<\mu<\left(2 \eta / k^{2}\right), 0 \leq \rho \tau<\nu$, where $\nu=1-\sqrt{1-\mu\left(2 \eta-\mu k^{2}\right)}$. And $\left\{\alpha_{n}\right\}$ and $\left\{\beta_{n}\right\}$ are sequences in $(0,1)$ satisfying the following conditions:

(a) $\lim _{n \rightarrow \infty} \alpha_{n}=0$ and $\sum_{n=1}^{\infty} \alpha_{n}=\infty$;

(b) $\lim _{n \rightarrow \infty}\left(\beta_{n} / \alpha_{n}\right)=0$;

(c) $\sum_{n=1}^{\infty}\left|\alpha_{n-1}-\alpha_{n}\right|<\infty$ and $\sum_{n=1}^{\infty}\left|\beta_{n-1}-\beta_{n}\right|<\infty$;

(d) $\liminf \operatorname{in}_{n \rightarrow \infty} r_{n}<\lim \sup _{n \rightarrow \infty} r_{n}<2 \varsigma$ and $\sum_{n=1}^{\infty} \mid r_{n-1}-$ $r_{n} \mid<\infty$.

Remark 12. Our method can be viewed as extension and improvement for some well-known results as follows

(i) The proposed method is an extension and improvement of the method of Wang and $\mathrm{Xu}$ [23] for finding the approximate element of the common set of solutions of a split equilibrium problem and a hierarchical fixed point problem in a real Hilbert space.

(ii) If the Lipschitzian mapping $U=f, F=I, \rho=\mu=$ 1 , we obtain an extension and improvement of the method of Yao et al. [14] for finding the approximate element of the common set of solutions of a split equilibrium problem and a hierarchical fixed point problem in a real Hilbert space.

(iii) The contractive mapping $f$ with a coefficient $\alpha \epsilon$ $[0,1)$ in other papers (see $[14,19,22,27])$ is extended to the cases of the Lipschitzian mapping $U$ with a coefficient constant $\gamma \in[0, \infty)$.

This shows that Algorithm 11 is quite general and unifying.

Lemma 13. Let $x^{*} \in \Lambda \cap F(T)$. Then $\left\{x_{n}\right\},\left\{u_{n}\right\}$, and $\left\{y_{n}\right\}$ are bounded.
Proof. Let $x^{*} \in \Lambda \cap F(T)$; we have $x^{*}=T_{r_{n}}^{F_{1}}\left(x^{*}\right)$ and $A x^{*}=$ $T_{r_{n}}^{F_{2}}\left(A x^{*}\right)$. Then

$$
\begin{aligned}
\left\|u_{n}-x^{*}\right\|^{2}= & \left\|T_{r_{n}}^{F_{1}}\left(x_{n}+\gamma A^{*}\left(T_{r_{n}}^{F_{2}}-I\right) A x_{n}\right)-x^{*}\right\|^{2} \\
= & \left\|T_{r_{n}}^{F_{1}}\left(x_{n}+\gamma A^{*}\left(T_{r_{n}}^{F_{2}}-I\right) A x_{n}\right)-T_{r_{n}}^{F_{1}}\left(x^{*}\right)\right\|^{2} \\
\leq & \left\|x_{n}+\gamma A^{*}\left(T_{r_{n}}^{F_{2}}-I\right) A x_{n}-x^{*}\right\|^{2} \\
= & \left\|x_{n}-x^{*}\right\|^{2}+\gamma^{2}\left\|A^{*}\left(T_{r_{n}}^{F_{2}}-I\right) A x_{n}\right\|^{2} \\
& +2 \gamma\left\langle x_{n}-x^{*}, A^{*}\left(T_{r_{n}}^{F_{2}}-I\right) A x_{n}\right\rangle \\
= & \left\|x_{n}-x^{*}\right\|^{2} \\
& +\gamma^{2}\left\langle\left(T_{r_{n}}^{F_{2}}-I\right) A x_{n}, A A^{*}\left(T_{r_{n}}^{F_{2}}-I\right) A x_{n}\right\rangle \\
& +2 \gamma\left\langle x_{n}-x^{*}, A^{*}\left(T_{r_{n}}^{F_{2}}-I\right) A x_{n}\right\rangle .
\end{aligned}
$$

From the definition of $L$, it follows that

$$
\begin{aligned}
\gamma^{2}\langle( & \left.\left.T_{r_{n}}^{F_{2}}-I\right) A x_{n}, A A^{*}\left(T_{r_{n}}^{F_{2}}-I\right) A x_{n}\right\rangle \\
& \leq L \gamma^{2}\left\langle\left(T_{r_{n}}^{F_{2}}-I\right) A x_{n},\left(T_{r_{n}}^{F_{2}}-I\right) A x_{n}\right\rangle \\
& =L \gamma^{2}\left\|\left(T_{r_{n}}^{F_{2}}-I\right) A x_{n}\right\|^{2} .
\end{aligned}
$$

It follows from (8) that

$$
\begin{aligned}
2 \gamma\left\langle x_{n}-x^{*}, A^{*}\left(T_{r_{n}}^{F_{2}}-I\right) A x_{n}\right\rangle \\
=2 \gamma\left\langle A\left(x_{n}-x^{*}\right),\left(T_{r_{n}}^{F_{2}}-I\right) A x_{n}\right\rangle \\
=2 \gamma\left\langle A\left(x_{n}-x^{*}\right)+\left(T_{r_{n}}^{F_{2}}-I\right) A x_{n}\right. \\
\left.\quad-\left(T_{r_{n}}^{F_{2}}-I\right) A x_{n},\left(T_{r_{n}}^{F_{2}}-I\right) A x_{n}\right\rangle \\
=2 \gamma\left(\left\langle T_{r_{n}}^{F_{2}} A x_{n}-A x^{*},\left(T_{r_{n}}^{F_{2}}-I\right) A x_{n}\right\rangle\right. \\
\left.\quad-\left\|\left(T_{r_{n}}^{F_{2}}-I\right) A x_{n}\right\|^{2}\right) \\
\leq 2 \gamma\left(\frac{1}{2}\left\|\left(T_{r_{n}}^{F_{2}}-I\right) A x_{n}\right\|^{2}-\left\|\left(T_{r_{n}}^{F_{2}}-I\right) A x_{n}\right\|^{2}\right) \\
=-\gamma\left\|\left(T_{r_{n}}^{F_{2}}-I\right) A x_{n}\right\|^{2} .
\end{aligned}
$$

Applying (36) and (35) to (34) and from the definition of $\gamma$, we get

$$
\begin{aligned}
\| u_{n}- & x^{*} \|^{2} \\
& \leq\left\|x_{n}-x^{*}\right\|^{2}+\gamma(L \gamma-1)\left\|\left(T_{r_{n}}^{F_{2}}-I\right) A x_{n}\right\|^{2} \\
& \leq\left\|x_{n}-x^{*}\right\|^{2} .
\end{aligned}
$$


Denote $V_{n}=\alpha_{n} \rho U\left(x_{n}\right)+\left(I-\alpha_{n} \mu F\right)\left(T\left(y_{n}\right)\right)$. Next, we prove that the sequence $\left\{x_{n}\right\}$ is bounded; without loss of generality we can assume that $\beta_{n} \leq \alpha_{n}$ for all $n \geq 1$. From (33), we have

$$
\begin{aligned}
& \left\|x_{n+1}-x^{*}\right\| \\
& =\left\|P_{C}\left[V_{n}\right]-P_{C}\left[x^{*}\right]\right\| \\
& \leq\left\|\alpha_{n} \rho U\left(x_{n}\right)+\left(I-\alpha_{n} \mu F\right)\left(T\left(y_{n}\right)\right)-x^{*}\right\| \\
& \leq \alpha_{n}\left\|\rho U\left(x_{n}\right)-\mu F\left(x^{*}\right)\right\| \\
& +\left\|\left(I-\alpha_{n} \mu F\right)\left(T\left(y_{n}\right)\right)-\left(I-\alpha_{n} \mu F\right) T\left(x^{*}\right)\right\| \\
& =\alpha_{n}\left\|\rho U\left(x_{n}\right)-\rho U\left(x^{*}\right)+(\rho U-\mu F)\left(x^{*}\right)\right\| \\
& +\left\|\left(I-\alpha_{n} \mu F\right)\left(T\left(y_{n}\right)\right)-\left(I-\alpha_{n} \mu F\right) T\left(x^{*}\right)\right\| \\
& \leq \alpha_{n} \rho \tau\left\|x_{n}-x^{*}\right\|+\alpha_{n}\left\|(\rho U-\mu F)\left(x^{*}\right)\right\| \\
& +\left(1-\alpha_{n} \nu\right)\left\|y_{n}-x^{*}\right\| \\
& \leq \alpha_{n} \rho \tau\left\|x_{n}-x^{*}\right\|+\alpha_{n}\left\|(\rho U-\mu F)\left(x^{*}\right)\right\| \\
& +\left(1-\alpha_{n} \nu\right)\left\|\beta_{n} S x_{n}+\left(1-\beta_{n}\right) u_{n}-x^{*}\right\| \\
& \leq \alpha_{n} \rho \tau\left\|x_{n}-x^{*}\right\|+\alpha_{n}\left\|(\rho U-\mu F)\left(x^{*}\right)\right\| \\
& +\left(1-\alpha_{n} \nu\right)\left(\beta_{n}\left\|S x_{n}-S x^{*}\right\|+\beta_{n}\left\|S x^{*}-x^{*}\right\|\right. \\
& \left.+\left(1-\beta_{n}\right)\left\|u_{n}-x^{*}\right\|\right) \\
& \leq \alpha_{n} \rho \tau\left\|x_{n}-x^{*}\right\|+\alpha_{n}\left\|(\rho U-\mu F)\left(x^{*}\right)\right\| \\
& +\left(1-\alpha_{n} \nu\right)\left(\beta_{n}\left\|S x_{n}-S x^{*}\right\|+\beta_{n}\left\|S x^{*}-x^{*}\right\|\right. \\
& \left.+\left(1-\beta_{n}\right)\left\|x_{n}-x^{*}\right\|\right) \\
& \leq \alpha_{n} \rho \tau\left\|x_{n}-x^{*}\right\|+\alpha_{n}\left\|(\rho U-\mu F)\left(x^{*}\right)\right\| \\
& +\left(1-\alpha_{n} \nu\right)\left(\beta_{n}\left\|x_{n}-x^{*}\right\|+\beta_{n}\left\|S x^{*}-x^{*}\right\|\right. \\
& \left.+\left(1-\beta_{n}\right)\left\|x_{n}-x^{*}\right\|\right) \\
& =\left(1-\alpha_{n}(\nu-\rho \tau)\right)\left\|x_{n}-x^{*}\right\| \\
& +\alpha_{n}\left\|(\rho U-\mu F)\left(x^{*}\right)\right\| \\
& +\left(1-\alpha_{n} \nu\right) \beta_{n}\left\|S x^{*}-x^{*}\right\| \\
& \leq\left(1-\alpha_{n}(\nu-\rho \tau)\right)\left\|x_{n}-x^{*}\right\| \\
& +\alpha_{n}\left\|(\rho U-\mu F)\left(x^{*}\right)\right\|+\beta_{n}\left\|S x^{*}-x^{*}\right\| \\
& \leq\left(1-\alpha_{n}(\nu-\rho \tau)\right)\left\|x_{n}-x^{*}\right\| \\
& +\alpha_{n}\left(\left\|(\rho U-\mu F)\left(x^{*}\right)\right\|+\left\|S x^{*}-x^{*}\right\|\right) \\
& =\left(1-\alpha_{n}(\nu-\rho \tau)\right)\left\|x_{n}-x^{*}\right\| \\
& +\frac{\alpha_{n}(\nu-\rho \tau)}{\nu-\rho \tau}\left(\left\|(\rho U-\mu F) x^{*}\right\|+\left\|S x^{*}-x^{*}\right\|\right) \\
& \leq \max \left\{\left\|x_{n}-x^{*}\right\|, \frac{1}{\nu-\rho \tau}\right. \\
& \left.\times\left(\left\|(\rho U-\mu F)\left(x^{*}\right)\right\|+\left\|S x^{*}-x^{*}\right\|\right)\right\},
\end{aligned}
$$

where the third inequality follows from Lemma 8.
By induction on $n$, we obtain $\left\|x_{n}-x^{*}\right\| \leq \max \left\{\| x_{0}-\right.$ $\left.x^{*} \|,(1 /(1-\rho))\left(\left\|(\rho U-\mu F) x^{*}\right\|+\left\|S x^{*}-x^{*}\right\|\right)\right\}$, for $n \geq 0$ and $x_{0} \in C$. Hence $\left\{x_{n}\right\}$ is bounded and, consequently, we deduce that $\left\{u_{n}\right\},\left\{y_{n}\right\},\left\{S\left(x_{n}\right)\right\},\left\{T\left(x_{n}\right)\right\},\left\{F\left(T\left(y_{n}\right)\right)\right\}$, and $\left\{U\left(x_{n}\right)\right\}$ are bounded.

Lemma 14. Let $x^{*} \in \Lambda \cap F(T)$ and $\left\{x_{n}\right\}$ the sequence generated by the Algorithm 11. Then one has

(a) $\lim _{n \rightarrow \infty}\left\|x_{n+1}-x_{n}\right\|=0$;

(b) the weak $w$-limit set $w_{w}\left(x_{n}\right) \subset F(T),\left(w_{w}\left(x_{n}\right)=\{x\right.$ : $\left.x_{n_{i}}-x\right\}$ ).

Proof. Since $u_{n}=T_{r_{n}}^{F_{1}}\left(x_{n}+\gamma A^{*}\left(T_{r_{n}}^{F_{2}}-I\right) A x_{n}\right)$ and $u_{n-1}=$ $T_{r_{n-1}}^{F_{1}}\left(x_{n-1}+\gamma A^{*}\left(T_{r_{n-1}}^{F_{2}}-I\right) A x_{n-1}\right)$ it follows from Lemma 5 that

$$
\begin{aligned}
& \left\|u_{n}-u_{n-1}\right\| \\
& \leq \| x_{n}-x_{n-1} \\
& +\gamma\left(A^{*}\left(T_{r_{n}}^{F_{2}}-I\right) A x_{n}-A^{*}\left(T_{r_{n-1}}^{F_{2}}-I\right) A x_{n-1}\right) \| \\
& +\left|1-\frac{r_{n-1}}{r_{n}}\right| \| T_{r_{n}}^{F_{1}}\left(x_{n}+\gamma A^{*}\left(T_{r_{n}}^{F_{2}}-I\right) A x_{n}\right) \\
& -\left(x_{n}+\gamma A^{*}\left(T_{r_{n}}^{F_{2}}-I\right) A x_{n}\right) \| \\
& \leq\left\|x_{n}-x_{n-1}-\gamma A^{*} A\left(x_{n}-x_{n-1}\right)\right\| \\
& +\gamma\|A\|\left\|T_{r_{n}}^{F_{2}} A x_{n}-T_{r_{n-1}}^{F_{2}} A x_{n-1}\right\| \\
& +\left|1-\frac{r_{n-1}}{r_{n}}\right| \| T_{r_{n}}^{F_{1}}\left(x_{n}+\gamma A^{*}\left(T_{r_{n}}^{F_{2}}-I\right) A x_{n}\right) \\
& -\left(x_{n}+\gamma A^{*}\left(T_{r_{n}}^{F_{2}}-I\right) A x_{n}\right) \| \\
& \leq\left(\left\|x_{n}-x_{n-1}\right\|^{2}-2 \gamma\left\|A\left(x_{n}-x_{n-1}\right)\right\|^{2}\right. \\
& \left.+\gamma^{2}\|A\|^{4}\left\|x_{n}-x_{n-1}\right\|^{2}\right)^{1 / 2} \\
& +\gamma\|A\|\left(\left\|A\left(x_{n}-x_{n-1}\right)\right\|\right. \\
& \left.+\left|1-\frac{r_{n-1}}{r_{n}}\right|\left\|T_{r_{n}}^{F_{2}} A x_{n}-A x_{n}\right\|\right) \\
& +\left|1-\frac{r_{n-1}}{r_{n}}\right| \| T_{r_{n}}^{F_{1}}\left(x_{n}+\gamma A^{*}\left(T_{r_{n}}^{F_{2}}-I\right) A x_{n}\right) \\
& -\left(x_{n}+\gamma A^{*}\left(T_{r_{n}}^{F_{2}}-I\right) A x_{n}\right) \| \\
& \leq\left(1-2 \gamma\|A\|^{2}+\gamma^{2}\|A\|^{4}\right)^{1 / 2}\left\|x_{n}-x_{n-1}\right\| \\
& +\gamma\|A\|^{2}\left\|x_{n}-x_{n-1}\right\| \\
& +\gamma\|A\|\left|1-\frac{r_{n-1}}{r_{n}}\right|\left\|T_{r_{n}}^{F_{2}} A x_{n}-A x_{n}\right\|
\end{aligned}
$$




$$
\begin{aligned}
& +\left|1-\frac{r_{n-1}}{r_{n}}\right| \| T_{r_{n}}^{F_{1}}\left(x_{n}+\gamma A^{*}\left(T_{r_{n}}^{F_{2}}-I\right) A x_{n}\right) \\
& \quad-\left(x_{n}+\gamma A^{*}\left(T_{r_{n}}^{F_{2}}-I\right) A x_{n}\right) \| \\
& =\left(1-\gamma\|A\|^{2}\right)\left\|x_{n}-x_{n-1}\right\| \\
& +\gamma\|A\|^{2}\left\|x_{n}-x_{n-1}\right\| \\
& +\gamma\|A\|\left|1-\frac{r_{n-1}}{r_{n}}\right|\left\|T_{r_{n}}^{F_{2}} A x_{n}-A x_{n}\right\| \\
& +\left|1-\frac{r_{n-1}}{r_{n}}\right| \| T_{r_{n}}^{F_{1}}\left(x_{n}+\gamma A^{*}\left(T_{r_{n}}^{F_{2}}-I\right) A x_{n}\right) \\
& \quad-\left(x_{n}+\gamma A^{*}\left(T_{r_{n}}^{F_{2}}-I\right) A x_{n}\right) \| \\
& +\left\|x_{n}-x_{n-1}\right\|+\gamma\|A\|\left|1-\frac{r_{n-1}}{r_{n}}\right|\left\|T_{r_{n}}^{F_{2}} A x_{n}-A x_{n}\right\| \\
& +\left|1-\frac{r_{n-1}}{r_{n}}\right| \| T_{r_{n}}^{F_{1}}\left(x_{n}+\gamma A^{*}\left(T_{r_{n}}^{F_{2}}-I\right) A x_{n}\right) \\
& \quad-\left(x_{n}+\gamma A^{*}\left(T_{r_{n}}^{F_{2}}-I\right) A x_{n}\right) \| \\
& =x_{n}-x_{n-1} \|+\left|\frac{r_{n}-r_{n-1}}{r_{n}}\right|\left(\gamma\|A\| \sigma_{n}+x_{n}\right),
\end{aligned}
$$

where $\sigma_{n}:=\left\|T_{r_{n}}^{F_{2}} A x_{n}-A x_{n}\right\|$ and $\chi_{n}:=\| T_{r_{n}}^{F_{1}}\left(x_{n}+\gamma A^{*}\left(T_{r_{n}}^{F_{2}}-\right.\right.$ I) $\left.A x_{n}\right)-\left(x_{n}+\gamma A^{*}\left(T_{r_{n}}^{F_{2}}-I\right) A x_{n}\right) \|$. Without loss of generality, let us assume that there exists a real number $\mu$ such that $r_{n}>$ $\mu>0$, for all positive integers $n$. Then we get

$$
\left\|u_{n-1}-u_{n}\right\| \leq\left\|x_{n-1}-x_{n}\right\|+\frac{1}{\mu}\left|r_{n-1}-r_{n}\right|\left(\gamma\|A\| \sigma_{n}+\chi_{n}\right) .
$$

From (33) and the above inequality, we get

$$
\begin{aligned}
\| y_{n}- & y_{n-1} \| \\
= & \| \beta_{n} S x_{n}+\left(1-\beta_{n}\right) u_{n} \\
& \quad-\left(\beta_{n-1} S x_{n-1}+\left(1-\beta_{n-1}\right) u_{n-1}\right) \| \\
= & \| \beta_{n}\left(S x_{n}-S x_{n-1}\right)+\left(\beta_{n}-\beta_{n-1}\right) S x_{n-1} \\
& +\left(1-\beta_{n}\right)\left(u_{n}-u_{n-1}\right)+\left(\beta_{n-1}-\beta_{n}\right) u_{n-1} \| \\
\leq & \beta_{n}\left\|x_{n}-x_{n-1}\right\|+\left(1-\beta_{n}\right)\left\|u_{n}-u_{n-1}\right\| \\
& +\left|\beta_{n}-\beta_{n-1}\right|\left(\left\|S x_{n-1}\right\|+\left\|u_{n-1}\right\|\right) \\
\leq & \beta_{n}\left\|x_{n}-x_{n-1}\right\|+\left(1-\beta_{n}\right) \\
& \times\left\{\left\|x_{n-1}-x_{n}\right\|+\frac{1}{\mu}\left|r_{n-1}-r_{n}\right|\left(\gamma\|A\| \sigma_{n}+\chi_{n}\right)\right\} \\
& +\left|\beta_{n}-\beta_{n-1}\right|\left(\left\|S x_{n-1}\right\|+\left\|u_{n-1}\right\|\right) \\
\leq & \left\|x_{n}-x_{n-1}\right\|+\frac{1}{\mu}\left|r_{n-1}-r_{n}\right|\left(\gamma\|A\| \sigma_{n}+\chi_{n}\right) \\
& +\left|\beta_{n}-\beta_{n-1}\right|\left(\left\|S x_{n-1}\right\|+\left\|u_{n-1}\right\|\right) .
\end{aligned}
$$

Next, we estimate

$$
\begin{aligned}
& \left\|x_{n+1}-x_{n}\right\| \\
& =\left\|P_{C}\left[V_{n}\right]-P_{C}\left[V_{n-1}\right]\right\| \\
& \leq \| \alpha_{n} \rho\left(U\left(x_{n}\right)-U\left(x_{n-1}\right)\right)+\left(\alpha_{n}-\alpha_{n-1}\right) \rho U\left(x_{n-1}\right) \\
& \quad+\left(I-\alpha_{n} \mu F\right)\left(T\left(y_{n}\right)\right)-\left(I-\alpha_{n} \mu F\right) T\left(y_{n-1}\right) \\
& \quad+\left(I-\alpha_{n} \mu F\right)\left(T\left(y_{n-1}\right)\right)-\left(I-\alpha_{n-1} \mu F\right)\left(T\left(y_{n-1}\right)\right) \| \\
& \leq \alpha_{n} \rho \tau\left\|x_{n}-x_{n-1}\right\|+\left(1-\alpha_{n} \nu\right)\left\|y_{n}-y_{n-1}\right\| \\
& +\left|\alpha_{n}-\alpha_{n-1}\right|\left(\left\|\rho U\left(x_{n-1}\right)\right\|+\left\|\mu F\left(T\left(y_{n-1}\right)\right)\right\|\right),
\end{aligned}
$$

where the second inequality follows from Lemma 8. From (41) and (42), we have

$$
\begin{aligned}
\| x_{n+1}- & x_{n} \| \\
\leq & \alpha_{n} \rho \tau\left\|x_{n}-x_{n-1}\right\|+\left(1-\alpha_{n} \nu\right) \\
& \times\left\{\left\|x_{n}-x_{n-1}\right\|+\frac{1}{\mu}\left|r_{n-1}-r_{n}\right|\left(\gamma\|A\| \sigma_{n}+x_{n}\right)\right. \\
& \left.+\left|\beta_{n}-\beta_{n-1}\right|\left(\left\|S x_{n-1}\right\|+\left\|u_{n-1}\right\|\right)\right\} \\
& +\left|\alpha_{n}-\alpha_{n-1}\right|\left(\left\|\rho U\left(x_{n-1}\right)\right\|+\left\|\mu F\left(T\left(y_{n-1}\right)\right)\right\|\right) \\
\leq & \left(1-(\nu-\rho \tau) \alpha_{n}\right)\left\|x_{n}-x_{n-1}\right\| \\
& +\frac{1}{\mu}\left|r_{n-1}-r_{n}\right|\left(\gamma\|A\| \sigma_{n}+\chi_{n}\right) \\
& +\left|\beta_{n}-\beta_{n-1}\right|\left(\left\|S x_{n-1}\right\|+\left\|u_{n-1}\right\|\right) \\
& +\left|\alpha_{n}-\alpha_{n-1}\right|\left(\left\|\rho U\left(x_{n-1}\right)\right\|+\left\|\mu F\left(T\left(y_{n-1}\right)\right)\right\|\right) \\
\leq & \left(1-(\nu-\rho \tau) \alpha_{n}\right)\left\|x_{n}-x_{n-1}\right\| \\
& +M\left(\frac{1}{\mu}\left|r_{n-1}-r_{n}\right|+\left|\beta_{n}-\beta_{n-1}\right|+\left|\alpha_{n}-\alpha_{n-1}\right|\right)
\end{aligned}
$$

where

$$
\begin{aligned}
M=\max & \left\{\sup _{n \geq 1}\left(\gamma\|A\| \sigma_{n}+\chi_{n}\right),\right. \\
& \sup _{n \geq 1}\left(\left\|S x_{n-1}\right\|+\left\|u_{n-1}\right\|\right), \\
& \left.\sup _{n \geq 1}\left(\left\|\rho U\left(x_{n-1}\right)\right\|+\left\|\mu F\left(T\left(y_{n-1}\right)\right)\right\|\right)\right\} .
\end{aligned}
$$

It follows from conditions (a)-(d) of Algorithm 11 and Lemma 9 that

$$
\lim _{n \rightarrow \infty}\left\|x_{n+1}-x_{n}\right\|=0 .
$$


The Scientific World Journal

7

Next, we show that $\lim _{n \rightarrow \infty}\left\|u_{n}-x_{n}\right\|=0$. Since $x^{*} \in \Lambda \cap F(T)$ by using (34) and (37), we obtain

$$
\begin{aligned}
& \left\|x_{n+1}-x^{*}\right\|^{2} \\
& =\left\langle P_{C}\left[V_{n}\right]-x^{*}, x_{n+1}-x^{*}\right\rangle \\
& =\left\langle P_{C}\left[V_{n}\right]-V_{n}, P_{C}\left[V_{n}\right]-x^{*}\right\rangle+\left\langle V_{n}-x^{*}, x_{n+1}-x^{*}\right\rangle \\
& \leq\left\langle\alpha_{n}\left(\rho U\left(x_{n}\right)-\mu F\left(x^{*}\right)+\left(I-\alpha_{n} \mu F\right)\left(T\left(y_{n}\right)\right)\right)\right. \\
& \left.-\left(I-\alpha_{n} \mu F\right)\left(T\left(x^{*}\right)\right), x_{n+1}-x^{*}\right\rangle \\
& =\left\langle\alpha_{n} \rho\left(U\left(x_{n}\right)-U\left(x^{*}\right)\right), x_{n+1}-x^{*}\right\rangle \\
& +\alpha_{n}\left\langle\rho U\left(x^{*}\right)-\mu F\left(x^{*}\right), x_{n+1}-x^{*}\right\rangle \\
& +\left\langle\left(I-\alpha_{n} \mu F\right)\left(T\left(y_{n}\right)\right)\right. \\
& \left.-\left(I-\alpha_{n} \mu F\right)\left(T\left(x^{*}\right)\right), x_{n+1}-x^{*}\right\rangle \\
& \leq \alpha_{n} \rho \tau\left\|x_{n}-x^{*}\right\|\left\|x_{n+1}-x^{*}\right\| \\
& +\alpha_{n}\left\langle\rho U\left(x^{*}\right)-\mu F\left(x^{*}\right), x_{n+1}-x^{*}\right\rangle \\
& +\left(1-\alpha_{n} \nu\right)\left\|y_{n}-x^{*}\right\|\left\|x_{n+1}-x^{*}\right\| \\
& \leq \frac{\alpha_{n} \rho \tau}{2}\left(\left\|x_{n}-x^{*}\right\|^{2}+\left\|x_{n+1}-x^{*}\right\|^{2}\right) \\
& +\alpha_{n}\left\langle\rho U\left(x^{*}\right)-\mu F\left(x^{*}\right), x_{n+1}-x^{*}\right\rangle \\
& +\frac{\left(1-\alpha_{n} \nu\right)}{2}\left(\left\|y_{n}-x^{*}\right\|^{2}+\left\|x_{n+1}-x^{*}\right\|^{2}\right) \\
& \leq \frac{\left(1-\alpha_{n}(\nu-\rho \tau)\right)}{2}\left\|x_{n+1}-x^{*}\right\|^{2} \\
& +\frac{\alpha_{n} \rho \tau}{2}\left\|x_{n}-x^{*}\right\|^{2} \\
& +\alpha_{n}\left\langle\rho U\left(x^{*}\right)-\mu F\left(x^{*}\right), x_{n+1}-x^{*}\right\rangle \\
& +\frac{\left(1-\alpha_{n} \nu\right)}{2}\left(\beta_{n}\left\|S x_{n}-x^{*}\right\|^{2}+\left(1-\beta_{n}\right)\left\|u_{n}-x^{*}\right\|^{2}\right) \\
& \leq \frac{\left(1-\alpha_{n}(\nu-\rho \tau)\right)}{2}\left\|x_{n+1}-x^{*}\right\|^{2} \\
& +\frac{\alpha_{n} \rho \tau}{2}\left\|x_{n}-x^{*}\right\|^{2} \\
& +\alpha_{n}\left\langle\rho U\left(x^{*}\right)-\mu F\left(x^{*}\right), x_{n+1}-x^{*}\right\rangle \\
& +\frac{\left(1-\alpha_{n} \nu\right) \beta_{n}}{2}\left\|S x_{n}-x^{*}\right\|^{2}+\frac{\left(1-\alpha_{n} \nu\right)\left(1-\beta_{n}\right)}{2} \\
& \times\left\{\left\|x_{n}-x^{*}\right\|^{2}+\gamma(L \gamma-1)\left\|\left(T_{r_{n}}^{F_{2}}-I\right) A x_{n}\right\|^{2}\right\},
\end{aligned}
$$

where the last inequality follows from (37), which implies that

$$
\begin{aligned}
\| x_{n+1}- & x^{*} \|^{2} \\
\leq & \frac{\alpha_{n} \rho \tau}{1+\alpha_{n}(\nu-\rho \tau)}\left\|x_{n}-x^{*}\right\|^{2} \\
& +\frac{2 \alpha_{n}}{1+\alpha_{n}(\nu-\rho \tau)}\left\langle\rho U\left(x^{*}\right)-\mu F\left(x^{*}\right), x_{n+1}-x^{*}\right\rangle \\
& +\frac{\left(1-\alpha_{n} \nu\right) \beta_{n}}{1+\alpha_{n}(\nu-\rho \tau)}\left\|S x_{n}-x^{*}\right\|^{2}+\frac{\left(1-\alpha_{n} \nu\right)\left(1-\beta_{n}\right)}{1+\alpha_{n}(\nu-\rho \tau)} \\
& \times\left\{\left\|x_{n}-x^{*}\right\|^{2}+\gamma(L \gamma-1)\left\|\left(T_{r_{n}}^{F_{2}}-I\right) A x_{n}\right\|^{2}\right\} \\
\leq & \frac{\alpha_{n} \rho \tau}{1+\alpha_{n}(\nu-\rho \tau)}\left\|x_{n}-x^{*}\right\|^{2} \\
& +\frac{2 \alpha_{n}}{1+\alpha_{n}(\nu-\rho \tau)}\left\langle\rho U\left(x^{*}\right)-\mu F\left(x^{*}\right), x_{n+1}-x^{*}\right\rangle \\
& +\left\|x_{n}-x^{*}\right\|^{2}+\frac{\left(1-\alpha_{n} \nu\right) \beta_{n}}{1+\alpha_{n}(\nu-\rho \tau)}\left\|S x_{n}-x^{*}\right\|^{2} \\
& -\frac{\left(1-\alpha_{n} \nu\right)\left(1-\beta_{n}\right) \gamma(1-L \gamma)}{1+\alpha_{n}(\nu-\rho \tau)}\left\|\left(T_{r_{n}}^{F_{2}}-I\right) A x_{n}\right\|^{2} .
\end{aligned}
$$

Then from the above inequality, we get

$$
\begin{aligned}
& \frac{\left(1-\alpha_{n} \nu\right)\left(1-\beta_{n}\right) \gamma(1-L \gamma)}{1+\alpha_{n}(\nu-\rho \tau)}\left\|\left(T_{r_{n}}^{F_{2}}-I\right) A x_{n}\right\|^{2} \\
& \leq \frac{\alpha_{n} \rho \tau}{1+\alpha_{n}(\nu-\rho \tau)}\left\|x_{n}-x^{*}\right\|^{2}+\frac{2 \alpha_{n}}{1+\alpha_{n}(\nu-\rho \tau)} \\
& \times\left\langle\rho U\left(x^{*}\right)-\mu F\left(x^{*}\right), x_{n+1}-x^{*}\right\rangle \\
&+\beta_{n}\left\|S x_{n}-x^{*}\right\|^{2}+\left\|x_{n}-x^{*}\right\|^{2}-\left\|x_{n+1}-x^{*}\right\|^{2} \\
& \leq \frac{\alpha_{n} \rho \tau}{1+\alpha_{n}(\nu-\rho \tau)}\left\|x_{n}-x^{*}\right\|^{2}+\frac{2 \alpha_{n}}{1+\alpha_{n}(\nu-\rho \tau)} \\
& \times\left\langle\rho U\left(x^{*}\right)-\mu F\left(x^{*}\right), x_{n+1}-x^{*}\right\rangle \\
&+\beta_{n}\left\|S x_{n}-x^{*}\right\|^{2}+\left(\left\|x_{n}-x^{*}\right\|+\left\|x_{n+1}-x^{*}\right\|\right) \\
& \times\left\|x_{n+1}-x_{n}\right\| .
\end{aligned}
$$

Since $\gamma(1-L \gamma)>0, \lim _{n \rightarrow \infty}\left\|x_{n+1}-x_{n}\right\|=0, \alpha_{n} \rightarrow 0$, and $\beta_{n} \rightarrow 0$, we obtain

$$
\lim _{n \rightarrow \infty}\left\|\left(T_{r_{n}}^{F_{2}}-I\right) A x_{n}\right\|=0 .
$$


8

The Scientific World Journal

Since $T_{r_{n}}^{F_{1}}$ is firmly nonexpansive, we have

$$
\begin{aligned}
\| u_{n}- & x^{*} \|^{2} \\
= & \left\|T_{r_{n}}^{F_{1}}\left(x_{n}+\gamma A^{*}\left(T_{r_{n}}^{F_{2}}-I\right) A x_{n}\right)-T_{r_{n}}^{F_{1}}\left(x^{*}\right)\right\|^{2} \\
\leq & \left\langle u_{n}-x^{*}, x_{n}+\gamma A^{*}\left(T_{r_{n}}^{F_{2}}-I\right) A x_{n}-x^{*}\right\rangle \\
= & \frac{1}{2}\left\{\left\|u_{n}-x^{*}\right\|^{2}+\left\|x_{n}+\gamma A^{*}\left(T_{r_{n}}^{F_{2}}-I\right) A x_{n}-x^{*}\right\|^{2}\right. \\
& \left.\quad-\left\|u_{n}-x^{*}-\left[x_{n}+\gamma A^{*}\left(T_{r_{n}}^{F_{2}}-I\right) A x_{n}-x^{*}\right]\right\|^{2}\right\} \\
= & \frac{1}{2}\left\{\left\|u_{n}-x^{*}\right\|^{2}+\left\|x_{n}+\gamma A^{*}\left(T_{r_{n}}^{F_{2}}-I\right) A x_{n}-x^{*}\right\|^{2}\right. \\
& \left.-\left\|u_{n}-x_{n}-\gamma A^{*}\left(T_{r_{n}}^{F_{2}}-I\right) A x_{n}\right\|^{2}\right\} \\
\leq & \frac{1}{2}\left\{\left\|u_{n}-x^{*}\right\|^{2}+\left\|x_{n}-x^{*}\right\|^{2}\right. \\
& \left.\quad-\left\|u_{n}-x_{n}-\gamma A^{*}\left(T_{r_{n}}^{F_{2}}-I\right) A x_{n}\right\|^{2}\right\} \\
= & \frac{1}{2}\left\{\left\|u_{n}-x^{*}\right\|^{2}+\left\|x_{n}-x^{*}\right\|^{2}\right. \\
-\left[\left\|u_{n}-x_{n}\right\|^{2}+\gamma^{2}\left\|A^{*}\left(T_{r_{n}}^{F_{2}}-I\right) A x_{n}\right\|^{2}\right. & \left.\left.-2 \gamma\left\langle u_{n}-x_{n}, A^{*}\left(T_{r_{n}}^{F_{2}}-I\right) A x_{n}\right\rangle\right]\right\}
\end{aligned}
$$

where the last inequality follows from (34) and (37). Hence, we get

$$
\begin{aligned}
\left\|u_{n}-x^{*}\right\|^{2} \leq & \left\|x_{n}-x^{*}\right\|^{2}-\left\|u_{n}-x_{n}\right\|^{2} \\
& +2 \gamma\left\|A u_{n}-A x_{n}\right\|\left\|\left(T_{r_{n}}^{F_{2}}-I\right) A x_{n}\right\| .
\end{aligned}
$$

From (46) and the above inequality, we have

$$
\begin{aligned}
\| x_{n+1}- & x^{*} \|^{2} \\
\leq & \frac{\left(1-\alpha_{n}(\nu-\rho \tau)\right)}{2}\left\|x_{n+1}-x^{*}\right\|^{2}+\frac{\alpha_{n} \rho \tau}{2}\left\|x_{n}-x^{*}\right\|^{2} \\
& +\alpha_{n}\left\langle\rho U\left(x^{*}\right)-\mu F\left(x^{*}\right), x_{n+1}-x^{*}\right\rangle \\
& +\frac{\left(1-\alpha_{n} \nu\right)}{2}\left(\beta_{n}\left\|S x_{n}-x^{*}\right\|^{2}+\left(1-\beta_{n}\right)\left\|u_{n}-x^{*}\right\|^{2}\right) \\
\leq & \frac{\left(1-\alpha_{n}(\nu-\rho \tau)\right)}{2}\left\|x_{n+1}-x^{*}\right\|^{2}+\frac{\alpha_{n} \rho \tau}{2}\left\|x_{n}-x^{*}\right\|^{2} \\
& +\alpha_{n}\left\langle\rho U\left(x^{*}\right)-\mu F\left(x^{*}\right), x_{n+1}-x^{*}\right\rangle+\frac{\left(1-\alpha_{n} \nu\right)}{2} \\
& \times\left\{\beta_{n}\left\|S x_{n}-x^{*}\right\|^{2}+\left(1-\beta_{n}\right)\right. \\
& \times\left(\left\|x_{n}-x^{*}\right\|^{2}-\left\|u_{n}-x_{n}\right\|^{2}\right. \\
& \left.\left.+2 \gamma\left\|A u_{n}-A x_{n}\right\|\left\|\left(T_{r_{n}}^{F_{2}}-I\right) A x_{n}\right\|\right)\right\},
\end{aligned}
$$

which implies that

$$
\begin{aligned}
\| x_{n+1} & -x^{*} \|^{2} \\
\leq & \frac{\alpha_{n} \rho \tau}{1+\alpha_{n}(\nu-\rho \tau)}\left\|x_{n}-x^{*}\right\|^{2}+\frac{2 \alpha_{n}}{1+\alpha_{n}(\nu-\rho \tau)} \\
& \times\left\langle\rho U\left(x^{*}\right)-\mu F\left(x^{*}\right), x_{n+1}-x^{*}\right\rangle \\
& +\frac{\left(1-\alpha_{n} \nu\right) \beta_{n}}{1+\alpha_{n}(\nu-\rho \tau)}\left\|S x_{n}-x^{*}\right\|^{2}+\frac{\left(1-\alpha_{n} \nu\right)\left(1-\beta_{n}\right)}{1+\alpha_{n}(\nu-\rho \tau)} \\
& \times\left\{\left\|x_{n}-x^{*}\right\|^{2}-\left\|u_{n}-x_{n}\right\|^{2}\right. \\
\leq & \frac{\alpha_{n} \rho \tau}{1+\alpha_{n}(\nu-\rho \tau)}\left\|x_{n}-x^{*}\right\|^{2}+\frac{2 \alpha_{n}}{1+\alpha_{n}(\nu-\rho \tau)} \\
& \times\left\langle\rho U\left(x^{*}\right)-\mu F\left(x^{*}\right), x_{n+1}-x^{*}\right\rangle \\
& +\frac{\left(1-\alpha_{n} \nu\right) \beta_{n}}{1+\alpha_{n}(\nu-\rho \tau)}\left\|S x_{n}-x^{*}\right\|^{2} \\
& +\left\|x_{n}-x^{*}\right\|^{2}+\frac{\left(1-\alpha_{n} \nu\right)\left(1-\beta_{n}\right)}{1+\alpha_{n}(\nu-\rho \tau)} \\
& \times\left\{-\left\|u_{n}-x_{n}\right\|^{2}+2 \gamma\left\|A u_{n}-A x_{n}\right\|\left\|\left(T_{r_{n}}^{F_{2}}-I\right) A x_{n}\right\|\right\} .
\end{aligned}
$$

Hence

$$
\begin{aligned}
& \frac{\left(1-\alpha_{n} \nu\right)\left(1-\beta_{n}\right)}{1+\alpha_{n}(\nu-\rho \tau)}\left\|u_{n}-x_{n}\right\|^{2} \\
& \leq \frac{\alpha_{n} \rho \tau}{1+\alpha_{n}(\nu-\rho \tau)}\left\|x_{n}-x^{*}\right\|^{2} \\
& +\frac{2 \alpha_{n}}{1+\alpha_{n}(\nu-\rho \tau)}\left\langle\rho U\left(x^{*}\right)-\mu F\left(x^{*}\right), x_{n+1}-x^{*}\right\rangle \\
& +\frac{\left(1-\alpha_{n} \nu\right) \beta_{n}}{1+\alpha_{n}(\nu-\rho \tau)}\left\|S x_{n}-x^{*}\right\|^{2} \\
& +\frac{2\left(1-\alpha_{n} \nu\right)\left(1-\beta_{n}\right) \gamma}{1+\alpha_{n}(\nu-\rho \tau)}\left\|A u_{n}-A x_{n}\right\|\left\|\left(T_{r_{n}}^{F_{2}}-I\right) A x_{n}\right\| \\
& +\left\|x_{n}-x^{*}\right\|^{2}-\left\|x_{n+1}-x^{*}\right\|^{2} \\
& \leq \frac{\alpha_{n} \rho \tau}{1+\alpha_{n}(\nu-\rho \tau)}\left\|x_{n}-x^{*}\right\|^{2} \\
& +\frac{2 \alpha_{n}}{1+\alpha_{n}(\nu-\rho \tau)}\left\langle\rho U\left(x^{*}\right)-\mu F\left(x^{*}\right), x_{n+1}-x^{*}\right\rangle \\
& +\frac{\left(1-\alpha_{n} \nu\right) \beta_{n}}{1+\alpha_{n}(\nu-\rho \tau)}\left\|S x_{n}-x^{*}\right\|^{2} \\
& +\frac{2\left(1-\alpha_{n} \nu\right)\left(1-\beta_{n}\right) \gamma}{1+\alpha_{n}(\nu-\rho \tau)}\left\|A u_{n}-A x_{n}\right\|\left\|\left(T_{r_{n}}^{F_{2}}-I\right) A x_{n}\right\| \\
& +\left(\left\|x_{n}-x^{*}\right\|+\left\|x_{n+1}-x^{*}\right\|\right)\left\|x_{n+1}-x_{n}\right\| \text {. }
\end{aligned}
$$


Since $\lim _{n \rightarrow \infty}\left\|x_{n+1}-x_{n}\right\|=0, \alpha_{n} \rightarrow 0, \beta_{n} \rightarrow 0$, and $\lim _{n \rightarrow \infty}\left\|\left(T_{r_{n}}^{F_{2}}-I\right) A x_{n}\right\|=0$, we obtain

$$
\lim _{n \rightarrow \infty}\left\|u_{n}-x_{n}\right\|=0
$$

Now, let $z \in \Lambda \cap F(T)$; since $T\left(x_{n}\right) \in C$, we have

$$
\begin{aligned}
&\left\|x_{n}-T\left(x_{n}\right)\right\| \\
& \leq\left\|x_{n}-x_{n+1}\right\|+\left\|x_{n+1}-T\left(x_{n}\right)\right\| \\
&=\left\|x_{n}-x_{n+1}\right\|+\left\|P_{C}\left[V_{n}\right]-P_{C}\left[T\left(x_{n}\right)\right]\right\| \\
& \leq\left\|x_{n}-x_{n+1}\right\| \\
&+\left\|\alpha_{n}\left(\rho U\left(x_{n}\right)-\mu F\left(T\left(y_{n}\right)\right)+T\left(y_{n}\right)-T\left(x_{n}\right)\right)\right\| \\
& \leq\left\|x_{n}-x_{n+1}\right\| \\
&+\alpha_{n}\left\|\rho U\left(x_{n}\right)-\mu F\left(T\left(y_{n}\right)\right)\right\|+\left\|y_{n}-x_{n}\right\| \\
& \leq\left\|x_{n}-x_{n+1}\right\|+\alpha_{n}\left\|\rho U\left(x_{n}\right)-\mu F\left(T\left(y_{n}\right)\right)\right\| \\
&+\left\|\beta_{n} S x_{n}+\left(1-\beta_{n}\right) u_{n}-x_{n}\right\| \\
& \leq\left\|x_{n}-x_{n+1}\right\|+\alpha_{n}\left\|\rho U\left(x_{n}\right)-\mu F\left(T\left(y_{n}\right)\right)\right\| \\
&+\beta_{n}\left\|S x_{n}-x_{n}\right\|+\left(1-\beta_{n}\right)\left\|u_{n}-x_{n}\right\| .
\end{aligned}
$$

Since $\lim _{n \rightarrow \infty}\left\|x_{n+1}-x_{n}\right\|=0, \alpha_{n} \rightarrow 0, \beta_{n} \rightarrow 0, \| \rho U\left(x_{n}\right)-$ $\mu F\left(T\left(y_{n}\right)\right) \|$, and $\left\|S x_{n}-x_{n}\right\|$ are bounded and $\lim _{n \rightarrow \infty} \| x_{n}-$ $u_{n} \|=0$, we obtain

$$
\lim _{n \rightarrow \infty}\left\|x_{n}-T\left(x_{n}\right)\right\|=0
$$

Since $\left\{x_{n}\right\}$ is bounded, without loss of generality, we can assume that $x_{n} \rightarrow x^{*} \in C$. It follows from Lemma 6 that $x^{*} \in F(T)$. Therefore $w_{w}\left(x_{n}\right) \subset F(T)$.

Theorem 15. The sequence $\left\{x_{n}\right\}$ generated by Algorithm 11 converges strongly to $z$, which is the unique solution of the variational inequality

$$
\langle\rho U(z)-\mu F(z), x-z\rangle \leq 0, \quad \forall x \in \Lambda \cap F(T) .
$$

Proof. Since $\left\{x_{n}\right\}$ is bounded $x_{n} \rightarrow w$ and from Lemma 14, we have $w \in F(T)$. Next, we show that $w \in \operatorname{EP}\left(F_{1}\right)$. Since $u_{n}=$ $T_{r_{n}}^{F_{1}}\left(x_{n}+\gamma A^{*}\left(T_{r_{n}}^{F_{2}}-I\right) A x_{n}\right)$, we have

$$
\begin{aligned}
F_{1}\left(u_{n}, y\right) & +\frac{1}{r_{n}}\left\langle y-u_{n}, u_{n}-x_{n}\right\rangle \\
& -\frac{1}{r_{n}}\left\langle y-u_{n}, \gamma A^{*}\left(T_{r_{n}}^{F_{2}}-I\right) A x_{n}\right\rangle \geq 0, \quad \forall y \in C .
\end{aligned}
$$

It follows from monotonicity of $F_{1}$ that

$$
\begin{aligned}
& -\frac{1}{r_{n}}\left\langle y-u_{n}, \gamma A^{*}\left(T_{r_{n}}^{F_{2}}-I\right) A x_{n}\right\rangle \\
& \quad+\frac{1}{r_{n}}\left\langle y-u_{n}, u_{n}-x_{n}\right\rangle \geq F_{1}\left(y, u_{n}\right), \quad \forall y \in C, \\
& -\frac{1}{r_{n_{k}}}\left\langle y-u_{n_{k}}, \gamma A^{*}\left(T_{r_{n_{k}}}^{F_{2}}-I\right) A x_{n_{k}}\right\rangle \\
& \quad+\left\langle y-u_{n_{k}}, \frac{u_{n_{k}}-x_{n_{k}}}{r_{n_{k}}}\right\rangle \geq F_{1}\left(y, u_{n_{k}}\right), \quad \forall y \in C .
\end{aligned}
$$

Since $\lim _{n \rightarrow \infty}\left\|u_{n}-x_{n}\right\|=0, \lim _{n \rightarrow \infty}\left\|\left(T_{r_{n}}^{F_{2}}-I\right) A x_{n}\right\|=0$, and $x_{n} \rightarrow w$, it easy to observe that $u_{n_{k}} \rightarrow w$. It follows by Assumption 3(iv) that $F_{1}(y, w) \leq 0$, for all $y \in C$.

For any $0<t \leq 1$ and $y \in C$, let $y_{t}=t y+(1-t) w$; we have $y_{t} \in C$. Then, from Assumptions 3((i) and (iv)), we have

$$
\begin{aligned}
0 & =F_{1}\left(y_{t}, y_{t}\right) \\
& \leq t F_{1}\left(y_{t}, y\right)+(1-t) F_{1}\left(y_{t}, w\right) \\
& \leq t F_{1}\left(y_{t}, y\right) .
\end{aligned}
$$

Therefore $F_{1}\left(y_{t}, y\right) \geq 0$. From Assumption 3(iii), we have $F_{1}(w, y) \geq 0$, which implies that $w \in \operatorname{EP}\left(F_{1}\right)$.

Next, we show that $A w \in \operatorname{EP}\left(F_{2}\right)$. Since $\left\{x_{n}\right\}$ is bounded and $x_{n} \rightarrow w$, there exists a subsequence $\left\{x_{n_{k}}\right\}$ of $\left\{x_{n}\right\}$ such that $x_{n_{k}} \rightarrow w$ and since $A$ is a bounded linear operator, $A x_{n_{k}} \rightarrow A w$. Now set $v_{n_{k}}=A x_{n_{k}}-T_{r_{n_{k}}}^{F_{2}} A x_{n_{k}}$. It follows from (49) that $\lim _{k \rightarrow \infty} v_{n_{k}}=0$ and $A x_{n_{k}}-v_{n_{k}}=T_{r_{n_{k}}}^{F_{2}} A x_{n_{k}}$. Therefore from the definition of $T_{r_{n_{k}}}^{F_{2}}$, we have

$$
\begin{aligned}
& F_{2}\left(A x_{n_{k}}-v_{n_{k}}, y\right) \\
& +\frac{1}{r_{n_{k}}}\left\langle y-\left(A x_{n_{k}}-v_{n_{k}}\right),\right. \\
& \left.\quad\left(A x_{n_{k}}-v_{n_{k}}\right)-A x_{n_{k}}\right\rangle \geq 0, \quad \forall y \in C .
\end{aligned}
$$

Since $F_{2}$ is upper semicontinuous in first argument, taking $\lim$ sup to above inequality as $k \rightarrow \infty$ and using Assumption 3(iv), we obtain

$$
F_{2}(A w, y) \geq 0 \quad \forall y \in C,
$$

which implies that $A w \in \operatorname{EP}\left(F_{2}\right)$ and hence $w \in \Lambda$.

Thus we have

$w \in \Lambda \cap F(T)$. 
Observe that the constants satisfy $0 \leq \rho \tau<\nu$ and

$$
\begin{aligned}
k & \geq \eta \\
& \Longleftrightarrow k^{2} \geq \eta^{2} \\
& \Longleftrightarrow 1-2 \mu \eta+\mu^{2} k^{2} \geq 1-2 \mu \eta+\mu^{2} \eta^{2} \\
& \Longleftrightarrow \sqrt{1-\mu\left(2 \eta-\mu k^{2}\right)} \geq 1-\mu \eta \\
& \Longleftrightarrow \mu \eta \geq 1-\sqrt{1-\mu\left(2 \eta-\mu k^{2}\right)} \\
& \Longleftrightarrow \mu \eta \geq \nu .
\end{aligned}
$$

Therefore from Lemma 7, the operator $\mu F-\rho U$ is $\mu \eta-\rho \tau$ strongly monotone, and we get the uniqueness of the solution of the variational inequality (58) and denote it by $z \in \Lambda \cap$ $F(T)$.

Next, we claim that $\lim \sup _{n \rightarrow \infty}\left\langle\rho U(z)-\mu F(z), x_{n}-z\right\rangle \leq$ 0 . Since $\left\{x_{n}\right\}$ is bounded, there exists a subsequence $\left\{x_{n_{k}}\right\}$ of $\left\{x_{n}\right\}$ such that

$$
\begin{aligned}
& \limsup _{n \rightarrow \infty}\left\langle\rho U(z)-\mu F(z), x_{n}-z\right\rangle \\
& \quad=\limsup _{k \rightarrow \infty}\left\langle\rho U(z)-\mu F(z), x_{n_{k}}-z\right\rangle \\
& \quad=\langle\rho U(z)-\mu F(z), w-z\rangle \leq 0 .
\end{aligned}
$$

Next, we show that $x_{n} \rightarrow z$. Consider

$$
\begin{aligned}
\left\|x_{n+1}-z\right\|^{2} & \left\langle P_{C}\left[V_{n}\right]-z, x_{n+1}-z\right\rangle \\
= & \left\langle P_{C}\left[V_{n}\right]-V_{n}, P_{C}\left[V_{n}\right]-z\right\rangle+\left\langle V_{n}-z, x_{n+1}-z\right\rangle \\
\leq & \left\langle\alpha_{n}\left(\rho U\left(x_{n}\right)-\mu F(z)\right)+\left(I-\alpha_{n} \mu F\right)\left(T\left(y_{n}\right)\right)\right. \\
& \left.\quad-\left(I-\alpha_{n} \mu F\right)(T(z)), x_{n+1}-z\right\rangle \\
\leq & \left\langle\alpha_{n} \rho\left(U\left(x_{n}\right)-U(z)\right), x_{n+1}-z\right\rangle \\
& +\alpha_{n}\left\langle\rho U(z)-\mu F(z), x_{n+1}-z\right\rangle \\
& +\left\langle\left(I-\alpha_{n} \mu F\right)\left(T\left(y_{n}\right)\right)-\left(I-\alpha_{n} \mu F\right)(T(z)), x_{n+1}-z\right\rangle \\
\leq & \alpha_{n} \rho \tau\left\|x_{n}-z\right\|\left\|x_{n+1}-z\right\| \\
& +\alpha_{n}\left\langle\rho U(z)-\mu F(z), x_{n+1}-z\right\rangle \\
& +\left(1-\alpha_{n} \nu\right)\left\|y_{n}-z\right\|\left\|x_{n+1}-z\right\| \\
\leq & \alpha_{n} \rho \tau\left\|x_{n}-z\right\|\left\|x_{n+1}-z\right\| \\
& +\alpha_{n}\left\langle\rho U(z)-\mu F(z), x_{n+1}-z\right\rangle \\
& +\left(1-\alpha_{n} \nu\right)\left\{\beta_{n}\left\|S x_{n}-S z\right\|+\beta_{n}\|S z-z\|\right. \\
\leq & \alpha_{n} \rho \tau\left\|x_{n}-z\right\|\left\|x_{n+1}-z\right\| \\
& \left.+\left(1-\beta_{n}\right)\left\|u_{n}-z\right\|\right\}\left\|x_{n+1}-z\right\| \\
&
\end{aligned}
$$

$$
\begin{aligned}
& \quad+\alpha_{n}\left\langle\rho U(z)-\mu F(z), x_{n+1}-z\right\rangle \\
& +\left(1-\alpha_{n} \nu\right)\left\{\beta_{n}\left\|x_{n}-z\right\|+\beta_{n}\|S z-z\|\right. \\
& \left.\quad+\left(1-\beta_{n}\right)\left\|x_{n}-z\right\|\right\}\left\|x_{n+1}-z\right\| \\
& =\left(1-\alpha_{n}(\nu-\rho \tau)\right)\left\|x_{n}-z\right\|\left\|x_{n+1}-z\right\| \\
& +\alpha_{n}\left\langle\rho U(z)-\mu F(z), x_{n+1}-z\right\rangle \\
& +\left(1-\alpha_{n} \nu\right) \beta_{n}\|S z-z\|\left\|x_{n+1}-z\right\| \\
& \leq \frac{1-\alpha_{n}(\nu-\rho \tau)}{2}\left(\left\|x_{n}-z\right\|^{2}+\left\|x_{n+1}-z\right\|^{2}\right) \\
& +\alpha_{n}\left\langle\rho U(z)-\mu F(z), x_{n+1}-z\right\rangle \\
& +\left(1-\alpha_{n} \nu\right) \beta_{n}\|S z-z\|\left\|x_{n+1}-z\right\|
\end{aligned}
$$

which implies that

$$
\begin{aligned}
&\left\|x_{n+1}-z\right\|^{2} \\
& \leq \frac{1-\alpha_{n}(\nu-\rho \tau)}{1+\alpha_{n}(\nu-\rho \tau)}\left\|x_{n}-z\right\|^{2} \\
&+\frac{2 \alpha_{n}}{1+\alpha_{n}(\nu-\rho \tau)}\left\langle\rho U\left(x_{n}\right)-\mu F(z), x_{n+1}-z\right\rangle \\
&+\frac{2\left(1-\alpha_{n} \nu\right) \beta_{n}}{1+\alpha_{n}(\nu-\rho \tau)}\|S z-z\|\left\|x_{n+1}-z\right\| \\
& \leq\left(1-\alpha_{n}(\nu-\rho \tau)\right)\left\|x_{n}-z\right\|^{2}+\frac{2 \alpha_{n}(\nu-\rho \tau)}{1+\alpha_{n}(\nu-\rho \tau)} \\
& \times\left\{\frac{1}{\nu-\rho \tau}\left\langle\rho U(z)-\mu F(z), x_{n+1}-z\right\rangle\right. \\
&\left.\quad+\frac{\left(1-\alpha_{n} \nu\right) \beta_{n}}{\alpha_{n}(\nu-\rho \tau)}\|S z-z\|\left\|x_{n+1}-z\right\|\right\} .
\end{aligned}
$$

Let $\gamma_{n}=\alpha_{n}(\nu-\rho \tau)$ and $\delta_{n}=\left(2 \alpha_{n}(\nu-\rho \tau) /(1+\right.$ $\left.\left.\alpha_{n}(\nu-\rho \tau)\right)\right)\left\{(1 /(\nu-\rho \tau))\left\langle\rho U(z)-\mu F(z), x_{n+1}-z\right\rangle+((1-\right.$ $\left.\left.\left.\alpha_{n} \nu\right) \beta_{n} / \alpha_{n}(\nu-\rho \tau)\right)\|S z-z\|\left\|x_{n+1}-z\right\|\right\}$.

Since

$$
\begin{gathered}
\sum_{n=1}^{\infty} \alpha_{n}=\infty, \\
\limsup _{n \rightarrow \infty}\left\{\frac{1}{\nu-\rho \tau}\left\langle\rho U(z)-\mu F(z), x_{n+1}-z\right\rangle\right. \\
\left.+\frac{\left(1-\alpha_{n} \nu\right) \beta_{n}}{\alpha_{n}(\nu-\rho \tau)}\|S z-z\|\left\|x_{n+1}-z\right\|\right\} \leq 0 .
\end{gathered}
$$

It follows that

$$
\sum_{n=1}^{\infty} \gamma_{n}=\infty, \quad \limsup _{n \rightarrow \infty} \frac{\delta_{n}}{\gamma_{n}} \leq 0 .
$$

Thus all the conditions of Lemma 9 are satisfied. Hence we deduce that $x_{n} \rightarrow z$. This completes the proof. 
Remark 16. In hierarchical fixed point problem (17), if $S=I-$ $(\rho U-\mu F)$, then we can get the variational inequality (58). In (58), if $U=0$, then we get the variational inequality $\langle F(z), x-$ $z\rangle \geq 0$, for all $x \in \Lambda \cap F(T)$, which is just the variational inequality studied by Suzuki [27] extending the common set of solutions of a system of variational inequalities, a split equilibrium problem, and a hierarchical fixed point problem.

\section{Conclusions}

In this paper, we suggest and analyze an iterative method for finding the approximate element of the common set of solutions of (15)-(16) and (17) in real Hilbert space, which can be viewed as a refinement and improvement of some existing methods for solving a split equilibrium problem and a hierarchical fixed point problem. Some existing methods (e.g., $[13,14,17-19,21-23])$ can be viewed as special cases of Algorithm 11. Therefore, the new algorithm is expected to be widely applicable.

\section{Conflict of Interests}

The author declares that there is no conflict of interests regarding the publication of this paper.

\section{Acknowledgment}

The author would like to thank Professor Omar Halli, Rector, Ibn Zohr University, for providing excellent research facilities.

\section{References}

[1] G. Crombez, "A geometrical look at iterative methods for operators with fixed points," Numerical Functional Analysis and Optimization, vol. 26, no. 2, pp. 157-175, 2005.

[2] G. Crombez, "A hierarchical presentation of operators with fixed points on Hilbert spaces," Numerical Functional Analysis and Optimization, vol. 27, pp. 259-277, 2006.

[3] H. Zhou, "Convergence theorems of fixed points for $\kappa$-strict pseudo-contractions in Hilbert spaces," Nonlinear Analysis, Theory, Methods and Applications, vol. 69, no. 2, pp. 456-462, 2008.

[4] S.-S. Chang, H. W. J. Lee, and C. K. Chan, "A new method for solving equilibrium problem fixed point problem and variational inequality problem with application to optimization," Nonlinear Analysis, Theory, Methods and Applications, vol. 70, no. 9, pp. 3307-3319, 2009.

[5] P. Katchang and P. Kumam, "A new iterative algorithm of solution for equilibrium problems, variational inequalities and fixed point problems in a Hilbert space," Journal of Applied Mathematics and Computing, vol. 32, no. 1, pp. 19-38, 2010.

[6] S. Plubtieng and R. Punpaeng, "A general iterative method for equilibrium problems and fixed point problems in Hilbert spaces," Journal of Mathematical Analysis and Applications, vol. 336, no. 1, pp. 455-469, 2007.

[7] X. Qin, M. Shang, and Y. Su, "A general iterative method for equilibrium problems and fixed point problems in Hilbert spaces," Nonlinear Analysis, Theory, Methods and Applications, vol. 69, no. 11, pp. 3897-3909, 2008.
[8] P. L. Combettes and S. A. Hirstoaga, "Equilibrium programming using proximal like algorithms," Mathematical Programming, vol. 78, pp. 29-41, 1997.

[9] Y. Censor, A. Gibali, and S. Reich, "Algorithms for the split variational inequality problem," Numerical Algorithms, vol. 59, no. 2, pp. 301-323, 2012.

[10] A. Moudafi, "Split Monotone Variational Inclusions," Journal of Optimization Theory and Applications, vol. 150, no. 2, pp. 275283, 2011.

[11] C. Byrne, Y. Censor, A. Gibali, and S. Reich, "Weak and strong convergence of algorithms for the split common null point problem," http://arxiv.org/abs/1108.5953.

[12] K. R. Kazmi and S. H. Rizvi, "Iterative approximation of a common solution of a split equilibrium problem, a variational inequality problem and a fixed point problem," Journal of the Egyptian Mathematical Society, vol. 21, pp. 44-51, 2013.

[13] G. Gu, S. Wang, and Y. J. Cho, "Strong convergence algorithms for hierarchical fixed points problems and variational inequalities," Journal of Applied Mathematics, vol. 2011, Article ID 164978, 17 pages, 2011.

[14] Y. Yao, Y. J. Cho, and Y.-C. Liou, "Iterative algorithms for hierarchical fixed points problems and variational inequalities," Mathematical and Computer Modelling, vol. 52, no. 9-10, pp. 1697$1705,2010$.

[15] A. Bnouhachem and M. A. Noor, "An iterative method for approximating the common solutions of a variational inequality, a mixed equilibrium problem and a hierarchical fixed point problem," Journal of Inequalities and Applications, vol. 490, pp. $1-25,2013$.

[16] A. Bnouhachem, "Algorithms of common solutions for a variational inequality, a split equilibrium problem and a hierarchical fixed point problem," Fixed Point Theory and Applications, vol. 2013, article 278, pp. 1-25, 2013.

[17] F. Cianciaruso, G. Marino, L. Muglia, and Y. Yao, "On a twosteps algorithm for hierarchical fixed point problems and variational inequalities," Journal of Inequalities and Applications, vol. 2009, Article ID 208692, 13 pages, 2009.

[18] P. E. Mainge and A. Moudafi, "Strong convergence of an iterative method for hierarchical fixed-point problems," Pacific Journal of Optimization, vol. 3, no. 3, pp. 529-538, 2007.

[19] G. Marino and H.-K. Xu, "A general iterative method for nonexpansive mappings in Hilbert spaces," Journal of Mathematical Analysis and Applications, vol. 318, no. 1, pp. 43-52, 2006.

[20] A. Moudafi, "Krasnoselski-Mann iteration for hierarchical fixed-point problems," Inverse Problems, vol. 23, no. 4, pp. 16351640, 2007.

[21] L.-C. Ceng, Q. H. Ansari, and J.-C. Yao, "Some iterative methods for finding fixed points and for solving constrained convex minimization problems," Nonlinear Analysis, Theory, Methods and Applications, vol. 74, no. 16, pp. 5286-5302, 2011.

[22] M. Tian, "A general iterative algorithm for nonexpansive mappings in Hilbert spaces," Nonlinear Analysis, Theory, Methods and Applications, vol. 73, no. 3, pp. 689-694, 2010.

[23] Y. Wang and W. Xu, "Strong convergence of a modified iterative algorithm for hierarchical fixed point problems and variational inequalities," Fixed Point Theory and Applications, vol. 2013, article 121, 9 pages, 2013.

[24] E. Blum and W. Oettli, "From optimization and variational inequalities to equilibrium problems," The Mathematics Student, vol. 63, pp. 123-145, 1994. 
[25] F. Cianciaruso, G. Marino, L. Muglia, and Y. Yao, "A hybrid projection algorithm for finding solutions of mixed equilibrium problem and variational inequality problem," Fixed Point Theory and Applications, vol. 2010, Article ID 383740, 19 pages, 2010.

[26] Y. Yao, Y.-C. Liou, and S. M. Kang, "Approach to common elements of variational inequality problems and fixed point problems via a relaxed extragradient method," Computers and Mathematics with Applications, vol. 59, no. 11, pp. 3472-3480, 2010.

[27] T. Suzuki, "Moudafi's viscosity approximations with MeirKeeler contractions," Journal of Mathematical Analysis and Applications, vol. 325, no. 1, pp. 342-352, 2007.

[28] H.-K. Xu, "Iterative algorithms for nonlinear operators," Journal of the London Mathematical Society, vol. 66, no. 1, pp. 240-256, 2002.

[29] G. L. Acedo and H.-K. Xu, "Iterative methods for strict pseudocontractions in Hilbert spaces," Nonlinear Analysis, Theory, Methods and Applications, vol. 67, no. 7, pp. 2258-2271, 2007. 


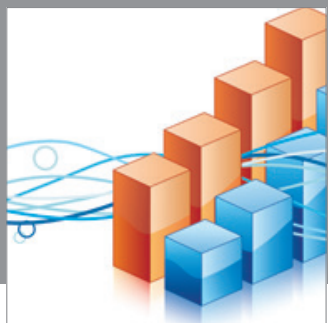

Advances in

Operations Research

mansans

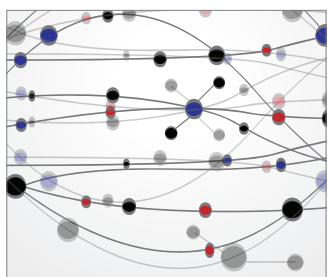

The Scientific World Journal
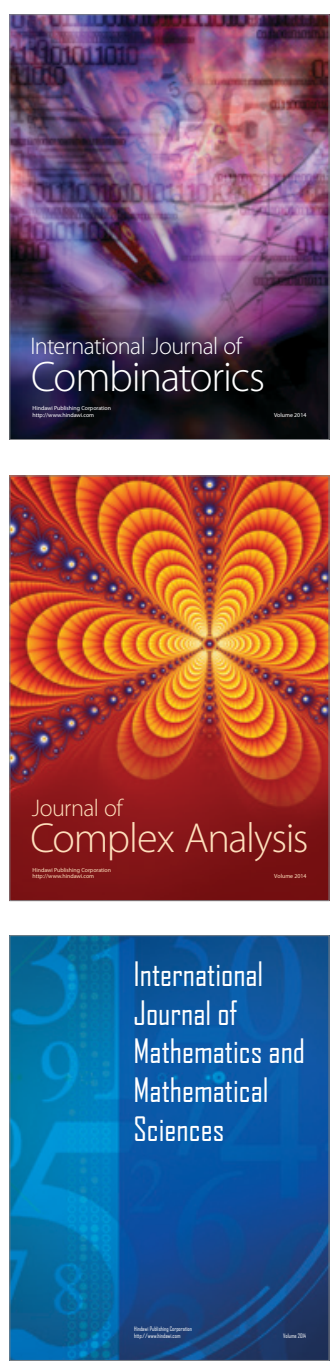
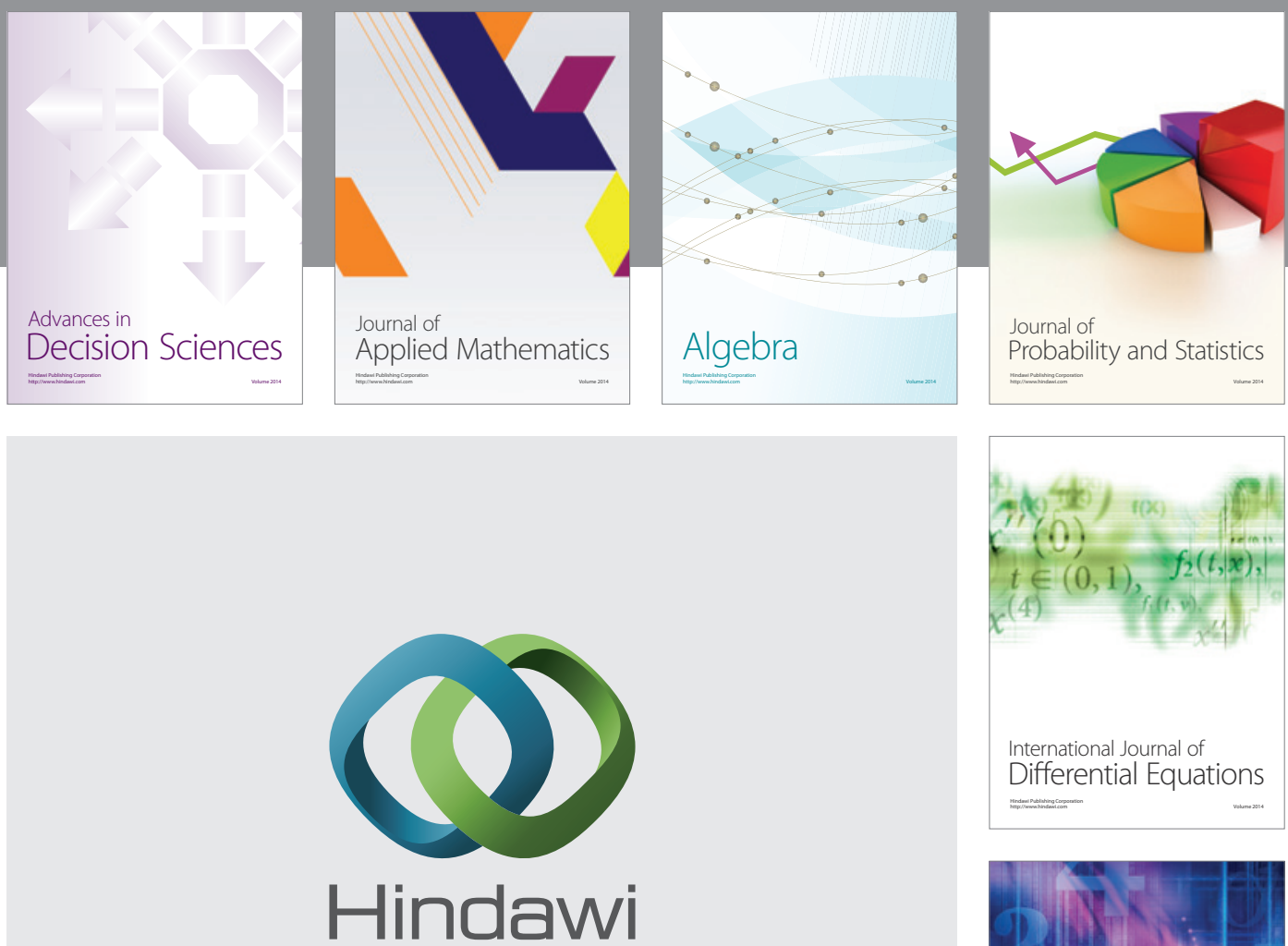

Submit your manuscripts at http://www.hindawi.com
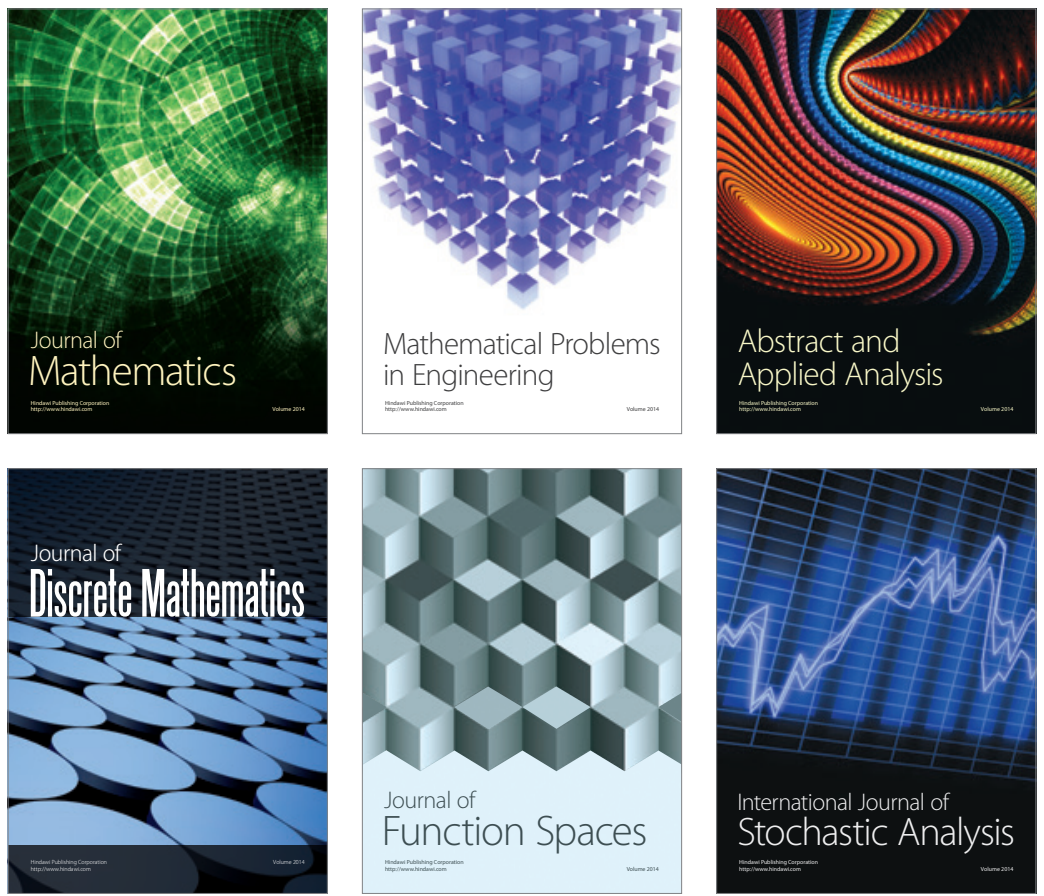

Journal of

Function Spaces

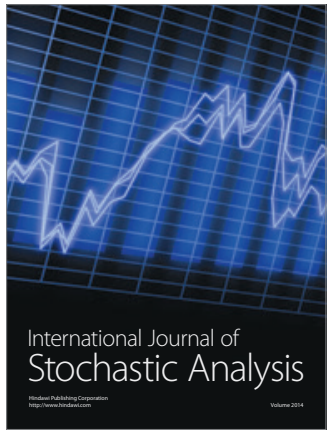

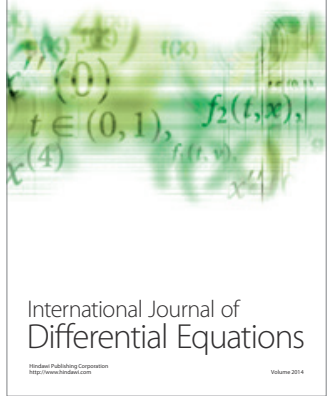
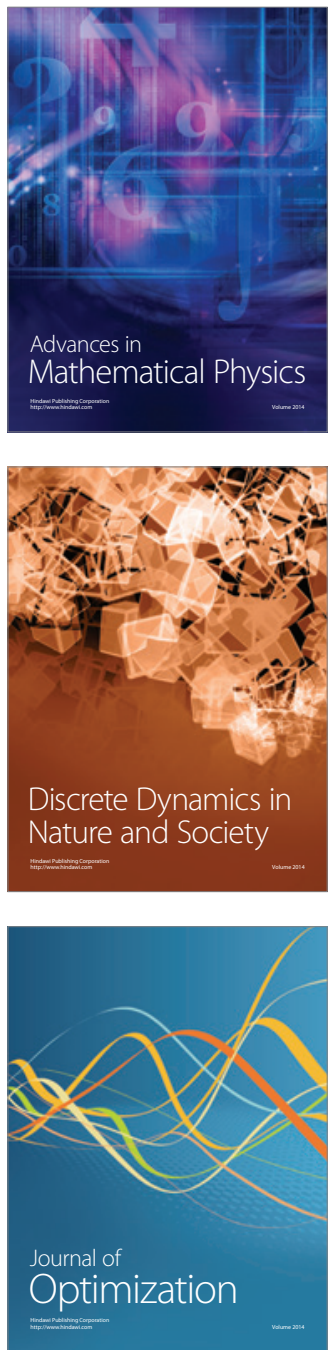